\title{
Prosody-Assisted Head-Driven Access to Spoken German Compounds
}

\author{
Frédéric Isel, Thomas C. Gunter, and Angela D. Friederici \\ Max Planck Institute of Cognitive Neuroscience
}

\begin{abstract}
Auditory processing of German 2-noun compound words was investigated with 328 participants in 4 experiments by monitoring semantic priming effects of the left constituents of the compound words. The authors demonstrated that there is no primacy of the left constituents in accessing auditorily presented German compound words in the mental lexicon. A clear priming effect of left constituents occurred only for compound words with a transparent right constituent that is the head of compound words in Germanic languages. The data suggest that the access to German compounds in the auditory domain involves 2 temporally overlapping routes: direct and decompositional. The prosodic structure (i.e., the duration) of the first morphemes of compound words appears to be a determining factor for activation of the decompositional route.
\end{abstract}

The nature of the lexical representations and the specificities of the cognitive processes that allow access to these representations have occupied a privileged position in research on the functional architecture of the mental lexicon. For morphologically complex words such as compound words in which morphemes are meaningful segments, the question of central interest is whether representations are word-based (Marslen-Wilson \& Welsh, 1978) or organized according to morphological aspects, with monosyllabic morphemes rather than whole words being the primary unit of representation (Taft \& Forster, 1975, 1976).

The study of lexical access to compound words containing two constituent words, both of which can exist independently as nouns, allows us to test these distinctive claims. By investigating the level of activation of each of the constituents during word perception, one can explore whether compound words have a decomposed representation (i.e., activation of one of their constituents) or a nondecomposed representation (i.e., no activation of the constituents) in the mental lexicon. The present study focuses on the role of the initial constituent during the auditory lexical access of two-noun German compound words (e.g., Gasthaus [guesthouse], containing Gast [guest] and Haus [house]). Our goal was to investigate whether this initial constituent serves as an access code that has to be activated during a sublexical stage of processing to allow a successful access to the compound word.

Frédéric Isel, Thomas C. Gunter, and Angela D. Friederici, Max Planck Institute of Cognitive Neuroscience, Leipzig, Germany.

This research was partially supported by a grant from the Fondation Fyssen (France) to Frédéric Isel and by a grant from the Max Planck Society (Germany). An early report of this research was presented at the Montreal Second International Conference on the Mental Lexicon in Montreal, Quebec, Canada (October 2000).

We thank Alexander Pollatsek and one anonymous reviewer as well as Kai Alter and Kerrie Elston-Guettler for helpful comments on the manuscript. We are also grateful to Juan Segui and Pienie Zwitserlood for fruitful discussions.

Correspondence concerning this article should be addressed to Frédéric Isel, Max Planck Institute of Cognitive Neuroscience, Stephanstrasse 1a, Postfach 500 355, Leipzig D-04103, Germany. E-mail: isel@cns.mpg.de

\section{Overview of Models}

Sequential discontinuous access models claim that lexical entries of mono- or polymorphemic words are accessed via representations of the first phonological syllable, irrespective of whether this syllable is a word (Taft \& Forster, 1976). ${ }^{1}$ Therefore, a primacy of monosyllabic left constituents of compound words is assumed. $^{2}$

In contrast, sequential continuous access theories refute the idea of an access code as a prerequisite for accessing the whole word. According to these models, the ongoing acoustic-phonetic information is, left to right, continuously mapped onto representations stored in the mental lexicon regardless of the morphological structure of the word (Butterworth, 1983; Marslen-Wilson, 1987).

\footnotetext{
${ }^{1}$ In the visual domain, Taft (1979) defines the basic orthographic syllabic structure (or BOSS) of a word as consisting of the first syllable, including the first vowel followed by all the consonants until an orthotactic violation disrupts the morphological structure of the word. The BOSS could be an access code to the lexicon. Thus, when the BOSS has more letters than the first phonological syllable (e.g., teap in teapot), the first phonological syllable is no longer the relevant unit of the access code. Therefore, no activation of the first monosyllabic constituents in compound words (e.g., tea in teapot) is expected. In our study, however, we refrained from formulating predictions with respect to the BOSS because we were working in the auditory modality rather than in the visual modality. As there is no direct correspondence between phonotactic and orthotactic violations for some consonantal clusters in German (e.g., the cluster /th/ in Gasthaus, which constitutes a phonotactic but not an orthotactic violation), we are skeptical as to the relevance of the BOSS for auditory processing. Moreover, over the four categories of compound words we used, the number of items with a BOSS that is larger than the first phonological syllable was similar (e.g., 10 compound words in each category).

${ }^{2}$ An alternative model might also assume two stages of processing, similar to Taft and Forster (1976), but determines not the phonological syllable but rather the head of the compound word, which contains the most relevant morphosyntactic-semantic information for the whole word, as being the relevant unit of the access code. The head of a compound word can be either the first or second constituent depending on the language under investigation. This assumption therefore implies a hierarchical access rather than a left-to-right access to right-headed compound words.
} 
Finally, dual-route models propose that discontinuous processing occurs for some words but not for others. These models postulate two possible routes of identifying complex words: (a) a decompositional route and (b) a direct route in which the word as a whole is accessed by a lexical entry. Either these two routes operate in parallel (Caramazza, Laudanna, \& Romani, 1988; Frauenfelder \& Schreuder, 1992; Schreuder \& Baayen, 1995), or their activation depends on the type of complex word (see the nonparallel model proposed by Niemi, Laine, \& Tuominen, 1994).

\section{Previous Research}

In the psycholinguistics literature, the various data obtained in visual word recognition using different experimental paradigms, such as cross-modal and unimodal priming, as well as in reading with different tasks (e.g., lexical decision, naming, eye movement monitoring, and progressive demasking) do not converge to an extent that they would allow a validation of one particular model (Andrews, 1986; Hyönä \& Pollatsek, 1998; Inhoff, Radach, \& Heller, 2000; Jarema, Busson, Nikolova, Tsapkini, \& Libben, 1999; Kehayia et al., 1999; Libben, 1994; Monsell, 1985; Pollatsek, Hyönä, \& Bertram, 2000; Sandra, 1990; Taft \& Forster, 1976; Zwitserlood, 1994). Using visually presented nonwords, Taft and Forster (1976) showed that the response latencies in a lexical decision task were influenced by the lexical status as well as by the frequency of the left constituents. The nonword footmilge (made up of a word and a nonword, respectively) took longer to classify as a nonword than did trowbreak (made up of a nonword and a word, respectively), suggesting that the initial fragment of English words, compared with a medial or a final fragment, contains the relevant information about the word status of the whole word. These results were interpreted to support the sequential search model introduced above.

Lima and Pollatsek (1983), in contrast, showed that nonword compounds in which both constituents formed real words (e.g., turntribe) took longer to reject in a visual lexical decision task than did those in which only the left constituent formed a word (e.g., hillsosk). This result suggests that the lexical status of the right constituent also influences the processing of nonword compound words. A similar pattern of results was observed by Andrews (1986) for real compound words.

Furthermore, lexical decision studies using a priming paradigm do not support a privileged status of left constituents, as they have revealed priming effects for both the left and the right constituents of compound words. Using visual-visual repetition priming, Monsell (1985, Experiment 8) found that butter and rope were able to prime butterfly and tightrope, respectively, both partially transparent compounds in regard to the right constituents (opaque-transparent; O-T). Sandra (1990) and Zwitserlood (1994), using visually presented two-noun Dutch compound words, showed a semantic priming effect for both the initial and the second constituent with fully transparent compound words (transparent-transparent; T-T), such as kerkorgel (church organ), but not with truly opaque compound words (opaque-opaque; O-O), such as klokhuis (apple core). Zwitserlood (Experiment 2) also found a semantic priming effect for both constituents of compound words that were partially transparent in regard to the left constituents (transparentopaque; T-O), such as drankorgel (booze organ). Moreover, Zwitserlood (Experiment 1) reported a repetition priming effect for both constituents of T-T, O-O, and T-O compound words. Similar results in the visual domain were obtained by Jarema et al. (1999) with French left-headed T-O, T-T, O-T, and O-O compound words (Experiment 1) and with right-headed Bulgarian T-T, O-T, and $\mathrm{T}-\mathrm{O}$ compound words (Experiment 2). ${ }^{3}$ The combined findings from these experiments suggest that at the level at which morphology is coded (either at the level of lexical form or at a separate level of representation between the level of lexical form and the semantic level), O-O compound words behave like any other type of real compound word, but at the semantic level, they behave just like monomorphemic words.

By monitoring eye movements during the reading of rightheaded Finnish two-noun T-T compound words, Hyönä and Pollatsek (1998, Experiment 2) showed that the gaze duration (i.e., the total fixation time on a word before the reader moves on to the next word) on the compound word was $100 \mathrm{~ms}$ shorter when there was a frequent initial morpheme. However, Pollatsek et al. (2000, Experiment 1) also demonstrated that the frequency of right constituents of T-T Finnish compound words had a large effect on the time taken to access the compound word. These findings support a decompositional model (Andrews, 1986; Sandra, 1990) or a hierarchical discontinuous model rather than a sequential left-toright model. Thus, the findings from studies conducted in the visual domain do not allow us to draw a final conclusion on the data at hand.

In the auditory domain, there is only one relevant study. It investigated the mental representation of T-T disyllabic spoken compound words in Mandarin Chinese using the differential frequency effect as a diagnostic tool (Zhou \& Marslen-Wilson, 1994). In three experiments in which morpheme frequency (MF) and syllable frequency (SF) of both the constituent morphemes and word frequency of the compound words were systematically varied, Zhou and Marslen-Wilson (1994) showed that the frequency of the entire disyllabic compound word is the dominant factor in determining response time in a lexical decision task and that this effect does not interact with variations in either the MF or the SF of either of the constituent morphemes, despite the salience and discriminability of morphemes and syllables in Chinese and despite the relative semantic transparency of the compound words under investigation. The authors therefore concluded that these results rather favor a whole-word representation model.

Taken together, what clearly emerges from the combined data collected in the visual domain and in the auditory domain is that a primacy of the first phonological syllable of compound words, as claimed by left-to-right discontinuous access theories, was not consistently reported for compound word processing. As has been demonstrated above, lexical access to compound words has mainly been investigated in the visual domain, therefore preventing us from being able to draw conclusions on the role of prosody. Prosody, however, has been attested as being a determining factor in access to the mental lexicon (see Cutler, Dahan, \& van Donselaar, 1997, for a review).

\footnotetext{
${ }^{3}$ Note, however, that no repetition priming effect was reported for O-O compound words for both left and right constituents, supporting the idea of a nondecomposability account for the fully opaque Bulgarian compound words.
} 


\section{The Present Study}

The present study focuses on the processing of compound words in the auditory domain, in which acoustic parameters of the left constituent already signal whether this morpheme is part of a compound. German is the language in which we conducted our experiments.

In German, the right constituent is the head of the compound word and is relevant for the assignment of syntactic gender. This particular morphological structure allowed us to investigate whether lexical access to compound words proceeds from left-toright as proposed by Taft and Forster (1976) or in a hierarchical manner via the head of the compound. An additional major factor concerning the representation of and access to morphologically complex words could be the transparency of the constituents. Because it is plausible that the lexical entry of a transparent compound word may be related, in some way, to the lexical entry of each of its constituents, we controlled for the factor of semantic transparency. This allowed us to isolate morphological factors from semantic factors. We thus used four categories of compound words that differed according to the presence or absence of a semantic link between the constituents and the compound word: T-T, O-O, O-T, and T-O. We explored lexical access to German compound words of the different types in the auditory domain using the cross-modal (auditory-visual) semantic priming paradigm (Tabossi, 1996) with a lexical decision task. ${ }^{4}$

Different models make various predictions for the different types of stimuli. For continuous models that assume an individual lexical entry for a compound word, access to a compound word does not depend on the processing of its constituents. In these models, neither left nor right constituents are relevant for lexical access. Discontinuous models assuming decomposition during lexical access make different predictions depending on additional assumptions they hold. Discontinuous models assuming left-toright processing and therefore a primacy of the left constituent predict priming effects for the left constituents in all conditions. Variants of this model incorporating the factor of transparency might predict priming effects for left constituents for those compound words in which the left constituent is transparent (i.e., T-T and $\mathrm{T}-\mathrm{O}$ ) but not for those in which the left constituent is opaque (i.e., O-O and O-T). Discontinuous models that assume a hierarchical access and thereby a primacy of the head of the compound word, in contrast, might predict priming effects for both right and left constituents in German compound words. Discontinuous models assuming primacy of the head but incorporating the factor of transparency might predict priming for transparent heads and for their left constituents (i.e., T-T and O-T) but not for opaque heads and their left constituents (i.e., O-O and T-O).

None of the different models explicitly discusses the role of prosodic information during lexical access to compound words in spoken language. Prosodic information available in the first constituent, however, already signals whether this word is part of a compound. An effective processing device might use this type of information during lexical access. Therefore, in the present study we varied the prosodic information available in the first constituent.

\section{Experiment 1}

In Experiment 1, we aimed to examine the processes engaged during lexical access to spoken two-noun German compound words. Because predictions of sequential continuous and discontinuous access theories largely diverge in explaining the role played by left constituents during lexical access to compound words, we decided to test the activation level of these constituents during the processing of the whole word.

Sequential continuous access theories postulating that access to compound words does not depend on the processing of one or two constituents (i.e., no morphological decomposition) predict no activation for the semantic associates of the left constituents irrespective of the type of compound word. In contrast, if access to the whole word depends on the preliminary activation of the left constituents (i.e., on morphological decomposition), as postulated by sequential discontinuous access theories, a facilitatory priming effect for the processing of targets semantically associated with the left constituents for each type of compound word should be expected. ${ }^{5}$ A larger priming effect should be measured for the transparent left constituents because of the additive effect of morphology and semantics.

\section{Method}

Participants. A group of 32 undergraduate students at the University of Leipzig ( 16 men and 16 women; mean age $=24.0$ years, range $=20-29$ years) were paid for their participation. They were native speakers of German with no reported auditory or (uncorrected) visual impairments. All participants were right-handed.

Materials and design. A pool of 160 two-noun German compound words were selected as primes. A search of the CELEX lexical database for German (Baayen, Piepenbrock, \& Gulikers, 1995) provided four categories of words using the criterion of transparency versus opacity for the constituents. We selected words from these categories to build our own categories: one consisting of $40 \mathrm{~T}-\mathrm{T}$ compound words (the two constituents were semantically associated with the compound word), one consisting of 40 $\mathrm{O}-\mathrm{O}$ compound words (no semantic association was present between the constituents and the whole word), one consisting of $40 \mathrm{~T}-\mathrm{O}$ compound words (only the left constituent presented a semantic association with the compound word), and one category of $40 \mathrm{O}-\mathrm{T}$ compound words (there was a semantic association only between the final constituents and the compound word). The left constituents were always monosyllabic words, whereas the right constituents were either monosyllables (67 words), disyllables ( 89 words) or trisyllables (4 words). For instance, the T-T compound word Gasthaus [guesthouse] contains the left constituent Gast [guest] and the right constituent (i.e., the head of the compound word) Haus [house]. Note that the left constituent refined the meaning of the final head constituent in T-T compound words.

To determine the semantic associates of the left constituents, we conducted a pretest of semantic association (Pretest 1) with 44 participants. Participants read each of the 160 monosyllabic left constituents and were instructed to give as rapidly as possible the first word that came to mind.

\footnotetext{
${ }^{4}$ The use of the semantic version of the priming paradigm increases the likelihood of tapping processes at a lexical rather than at a sublexical level (Slowiaczek, Nusbaum, \& Pisoni, 1987).

${ }^{5}$ According to a model of spreading activation (Collins \& Loftus, 1975), if primes and targets present an associative relation in the lexicon, then the activation generated in the prime representation will spread along associative pathways to the target, thus facilitating the recognition of the target. This facilitation should be attested by a positive priming effect: by shorter lexical decision latencies for the processing of the targets in the related condition than in the unrelated condition. In contrast, if primes and targets do not present any associative relation in the lexicon, no facilitatory priming effect should be expected for the processing of the targets.
} 
Only the semantic associates being given in at least $25 \%$ of the cases were kept (66\% of the constituents). For the $34 \%$ of the constituents for which the association with a word was difficult to establish because of many potential associates (on average, five associates), we conducted a second pretest (Pretest 2). Twenty participants who did not take part in Pretest 1 were instructed to read each of the constituents carefully. Then, we instructed them to write down which word was the best candidate among the proposed associates that had been determined by Pretest 1 . On average, the selected visual targets we used were chosen by the participants in $30 \%$ $(S E M=1.8)$ of the cases as being semantically associated with the primes. ${ }^{6}$ The percentage of semantic association did not significantly differ between the four categories of compound words $(F<1)$.

Moreover, the number of letters for targets was controlled. On average, the visual targets had five letters. Visual targets with more than seven letters ( $M \pm 2 S D ; 3.7 \%$ of targets) were replaced with synonyms. All targets were nouns of medium frequency (75.6 occurrences per million, $S E M=11.2$ ), with the exception of 10 nouns that were of high frequency ( $>200$ occurrences per million).

We next investigated whether the selected semantic associates could be primed by the compound words themselves. A test of this was to assess whether the compound word and left constituent shared the same semantic associates. Thus, we conducted Pretest 3 to determine the semantic associate for each of the 160 compound words. Forty-four participants who had not taken part in the previous two pretests read the 160 compound words and were instructed to give as rapidly as possible the first word that came to mind. We determined for each compound word the stronger semantic associate. For each compound word, we compared the two semantic associates respectively given for the left constituents (Pretests 1 and 2) and for the compound words (Pretest 3 ). In each compound word category, six semantic associates were found to be identical or semantically very close. We discarded from the pool of critical items these 24 compound words (6 words $\times 4$ categories). Thus, the final experimental pool contained 136 compound words ( 34 words in each of the four categories). The 136 compound words were low-frequency nouns: Their mean frequency was three $(S E M=0.9)$ occurrences per million. Left and right constituents were nouns of medium frequency, with $71(S E M=12.4)$ and 54 $(S E M=7.9)$ occurrences per million, respectively.

In the related test condition, a participant heard the prime word (e.g., Gasthaus [guesthouse]) and had to make a lexical decision on the visually presented probe (e.g., Besuch [visit]). In the unrelated control condition, the same probes were preceded by 136 polysyllabic compound words that were matched on length, syllabic structure, and frequency with the primes in the related condition (e.g., Schrankfach [compartment] was presented before Besuch [visit]). In addition, we selected 408 filler pairs presenting neither a morphological nor a semantic link between prime and target. They were polysyllabic compound words that contained a monosyllabic left constituent and a mono-, bi-, or trisyllabic right constituent. A total of 408 items were selected to serve as visual targets. Among them were 136 real monosyllabic words (five letters on average) and 272 pseudowords (five letters on average). Pseudowords were created from a pool of real words by changing a single letter in either the initial, medial, or final position. Items in prime and in probe positions were paired to create 136 unrelated word-word pairs and 272 unrelated word-pseudoword pairs.

In each list, 136 critical prime-target pairs were presented: 68 related pairs and 68 unrelated pairs. In each list, the 68 related trials and the 68 unrelated trials were pseudorandomly interspersed with the 408 filler pairs. The following constraints were respected: First, related pairs were not presented in more than three consecutive trials. Second, no more than four pairs with word or nonword targets were presented in succession. Third, no experimental stimulus was included among the first three stimuli of a block. Finally, the few pairs that, as a visual target, did present a repetition of left constituents of critical compound words were placed at intervals of at least 80 items (e.g., Kuhstall-Wiese [cow house-meadow] and Rindvieh-Kuh [cattle-cow]).
By means of a Latin square design, two experimental lists were created such that related and unrelated pairs were balanced across the lists. For each list, two sequences were constructed, the second in the reverse order of the first. Half the participants were tested on each sequence. Each target was presented under both priming conditions, but no participant heard the same prime twice nor saw the same target twice, thus avoiding stimulus repetition effects. Following the standard procedure used in priming experiments with a Latin square design, 2 participants (i.e., 1 per list) presented with complementary prime-target pairs were considered as 1 supersubject (Davis, Marslen-Wilson, \& Gaskell, 2002; Frost, Deutsch, \& Forster, 2000; Laudanna, Badecker, \& Caramazza, 1989), that is, a single point for statistical analysis. The 24 participants tested in the experiment thus formed 12 supersubjects.

Half of the visual targets (i.e., 272 targets) were German words, and half were pseudowords (i.e., 272 targets). The proportion of related pairs was $12.5 \%$ to prevent participants from adopting a response strategy (Sandra, 1990). Each list consisted of four blocks containing 136 pairs each. Thus, each participant processed 544 prime-target pairs.

Procedure. Auditory primes were recorded by a female native speaker of German in an acoustically shielded room. They were digitized at $44 \mathrm{kHz}$ with 16-bit resolution, equated for root mean square amplitude, and stored on the hard disk of a PC for presentation to participants. At the beginning of each trial, a fixation point appeared at the center of a computer monitor to draw the participant's attention to the position of the upcoming target word. At the acoustic offset of the prime, the fixation point disappeared and the visual target was presented in uppercase letters. The visual target remained on the screen until the participant responded. When the target had an even number of letters, the distribution of letters was symmetrical about the fixation point. For an odd number of letters, the fixation point corresponded to the median letter. The interstimulus interval was set at $0 \mathrm{~ms}$; the intertrial interval was $2 \mathrm{~s}$. The interblock interval was not defined, as participants decided themselves when they wanted to resume.

Participants were tested individually in a quiet room. Auditory stimuli were presented binaurally via headphones at a comfortable listening level. Participants were instructed to indicate as accurately and quickly as possible whether the visual target was a word or a nonword. They responded by pressing one of two buttons on a response box using their dominant hand. Responses and reaction times (RTs) were recorded for each trial and stored on a computer hard disk. A counter module was started at the onset of the visual target to register RTs. Time-out was set at $200 \mathrm{~ms}$ and at 1,500 $\mathrm{ms}$; if the participant responded before $200 \mathrm{~ms}$ or after $1,500 \mathrm{~ms}$, the response was coded as missing. Twenty practice trials were presented prior to the experimental trials. Each session lasted about $60 \mathrm{~min}$.

We averaged the RTs for correct responses in the eight experimental conditions across participants and across items. RTs that were outside a range of two standard deviations above and below the mean for each participant were excluded from the statistical analysis. Trials on which an error occurred were discarded. This procedure was repeated in all of the following experiments.

\section{Results}

The question of central interest in Experiment 1 was whether the left constituent of a compound word is activated during the pro-

\footnotetext{
${ }^{6}$ In two cross-modal semantic priming experiments with a lexical decision task additionally conducted for the present study, we showed that the semantic associates used as visual targets of the left constituents (e.g., Besuch [visit]) were able to be primed by the left constituents when they were presented as individual auditory primes (e.g., Gast [guest] primed Besuch [visit]): T-T $=28 \mathrm{~ms} ; \mathrm{O}-\mathrm{O}=29 \mathrm{~ms} ; \mathrm{O}-\mathrm{T}=31 \mathrm{~ms} ; \mathrm{T}-\mathrm{O}=21 \mathrm{~ms}$ They were not able to be primed by the right constituents of the compound words (e.g., Haus [house] did not prime Besuch [visit]): T-T $=-1 \mathrm{~ms}$; $\mathrm{O}-\mathrm{O}=3 \mathrm{~ms} ; \mathrm{O}-\mathrm{T}=-4 \mathrm{~ms}$; $\mathrm{T}-\mathrm{O}=-5 \mathrm{~ms}$.
} 
cessing of the whole word. The relevant data are presented in Table 1 . We subjected the results to a four-way analysis of variance (ANOVA) in which relatedness (related or unrelated), left constituent (transparent or opaque), and right constituent (transparent or opaque) were considered as within-subjects factors and in which word list (List 1 or List 2) was considered as a betweensubjects factor. ${ }^{7}$ The word list factor was introduced merely to extract any variance due to the counterbalancing. A significance level of $\alpha=.05$ was used for all statistical tests.

The word list factor was not significant in both participant and item analyses $(F \mathrm{~s}<1)$, indicating that the counterbalancing of items in the two experimental lists did not introduce variance in the results. Thus, for each of the eight experimental conditions, we collapsed complementary data of the two experimental lists.

The mean lexical decision latency was significantly shorter for the related condition $(490 \mathrm{~ms}, S E M=10.8)$ than for the unrelated condition $(501 \mathrm{~ms}, S E M=11), F_{1}(1,15)=27.75, M S E=135.58$, and $F_{2}(1,33)=18.66, M S E=470.27$. A significant interaction between relatedness and right constituent was found, $F_{1}(1$, $15)=6.00, M S E=187.89$, and $F_{2}(1,33)=5.54, M S E=691.24$, indicating that the difference between the related and the unrelated conditions was larger when the right constituents of the compound words were transparent (i.e., $17 \mathrm{~ms}$ ) than when they were opaque (i.e., $5 \mathrm{~ms}$; see Table 2).

Subsequent post hoc tests revealed that only the 17-ms facilitatory priming effect measured for the compound words with a

Table 1

Reaction Times (RTs; in Milliseconds) and Percentages of Error for Lexical Decisions to Target Words Associated With the Left Constituents for Each Category of Compound Word in Each Condition for Each Experiment

\begin{tabular}{lllll}
\hline Condition & T-T & O-O & O-T & T-O \\
\hline
\end{tabular}

Experiment $1\left([\mathrm{C} 1-\mathrm{C} 2]^{\mathrm{a}}\right)$

\begin{tabular}{lllll} 
Related & $493(3.1)$ & $488(2.1)$ & $491(1.3)$ & $487(2.0)$ \\
Unrelated & $513(3.6)$ & $490(2.0)$ & $505(2.1)$ & $494(1.8)$ \\
\hline
\end{tabular}

Experiment $2\left(\mathrm{C}^{\mathrm{a}}-\mathrm{C} 2\right)$

\begin{tabular}{lllll} 
Related & $505(4.8)$ & $504(3.6)$ & $499(3.8)$ & $503(4.2)$ \\
Unrelated & $512(4.4)$ & $502(5.1)$ & $498(4.9)$ & $508(4.6)$ \\
\hline
\end{tabular}

Experiment $3\left(\left[\mathrm{C} 1^{\mathrm{b}}-\mathrm{C} 2\right]^{\mathrm{a}}\right)$

\begin{tabular}{lllll} 
Related & $543(4.9)$ & $507(4.9)$ & $524(3.6)$ & $517(2.1)$ \\
Unrelated & $558(6.4)$ & $526(4.1)$ & $530(3.2)$ & $527(1.9)$ \\
\hline
\end{tabular}

Experiment $4 \mathrm{~A}\left(\left[\mathrm{C} 1-\mathrm{C} 2^{\mathrm{c}}\right]^{\mathrm{a}}\right)$

\begin{tabular}{lcccc} 
Related & $535(4.6)$ & $513(2.8)$ & $522(3.5)$ & $515(3.6)$ \\
Unrelated & $537(6.6)$ & $514(3.1)$ & $524(3.3)$ & $519(4.4)$ \\
\hline \multicolumn{5}{c}{ Experiment $4 \mathrm{~B}\left([\mathrm{C} 1-\mathrm{C} 2]^{\mathrm{a}}\right)^{\mathrm{d}}$} \\
Related & $530(6.2)$ & $502(3.1)$ & $513(3.6)$ & $511(4.8)$ \\
Unrelated & $553(7.1)$ & $503(3.5)$ & $529(4.5)$ & $518(6.5)$ \\
\hline
\end{tabular}

Note. $\quad \mathrm{T}-\mathrm{T}=$ transparent-transparent; $\mathrm{O}-\mathrm{O}=$ opaque-opaque; $\mathrm{O}-\mathrm{T}=$ opaque-transparent; $\mathrm{T}-\mathrm{O}=$ transparent-opaque; $\mathrm{C} 1=$ left constituent; $\mathrm{C} 2=$ right constituent.

${ }^{\mathrm{a}}$ Visual targets appeared in the speech signal. ${ }^{\mathrm{b}}$ Cues of coarticulation were absent. ${ }^{\mathrm{c}} \mathrm{C} 2$ was noise. ${ }^{\mathrm{d}}$ Replication of Experiment 1. transparent head was significant across participants, $F_{1}(1$, $15)=50.94, M S E=44.73$, and across items, $F_{2}(1,33)=17.09$, $M S E=366.90 .^{8}$ Descriptive statistics showed that this significant priming effect accounted for $77 \%(\eta=.77)$ of the variance of the dependent measure across participants and for $34 \%(\eta=.34)$ of the variance across items, indicating that this effect is large. In contrast, the nonsignificant priming effect reported for compound words with an opaque head (i.e., $5 \mathrm{~ms}$ ) accounted for only $10 \%$ $(\eta=.10)$ of the variance across participants and for only $3 \%(\eta=$ $.03)$ of the variance across items, reflecting that this effect is small. The two facilitatory priming effects (i.e., $17 \mathrm{~ms}$ vs. $5 \mathrm{~ms}$; see Table 2) did differ significantly, $F_{1}(1,15)=6.31, M S E=190.23$, and $F_{2}(1,33)=6.49, M S E=678.16$. Furthermore, relatedness had no effect on errors (related $=2.1 \%$, unrelated $=2.4 \% ; F \mathrm{~s}<1$ ).

To ensure the robustness of the priming effects, we tested whether the 136 selected compound words were correctly distributed over the four categories according to the criterion of transparency versus opacity for their constituents. A posttest of semantic relatedness was conducted with 28 participants to control the semantic association between the compound words and their constituents. The results showed that the 68 weak prototypes behaved just like the 68 strong prototypes. For this reason, we no longer used this criterion to split our pool of items into two subgroups. Further experiments were thus conducted using the total pool of 136 compound words.

Finally, to ensure that there was no possible confounding between the factor of right constituent (transparent or opaque) and the syllabic structure of the right constituent (monosyllable or disyllable), we conducted a four-way ANOVA with the factors of relatedness (related or unrelated), left constituent (transparent or opaque), right constituent (transparent or opaque), and syllabic structure of the right constituent (monosyllable or disyllable). Because neither the main effect of syllabic structure of the right constituent nor the Relatedness $\times$ Syllabic Structure of the Right Constituent interaction were significant across either participants or items, the hypothesis of a possible confounding between the factors of right constituent and syllabic structure of the right constituent can be rejected.

\section{Discussion}

Experiment 1 yielded two interesting results: First, facilitatory effects were not observed for the four categories of compound words. Second and more important, the left constituents regardless of transparency showed priming only when the right constituent

\footnotetext{
${ }^{7}$ For all experiments, we report only significant main effects and significant interactions to simplify the presentation of the Results sections.

${ }^{8}$ To determine whether the facilitatory priming effect was attributable to the activation of the left constituents only or to the activation of both the left constituents and the compound words (an additive effect), we ensured that the priming effect obtained in Experiment 1 was not significantly larger than the priming effect obtained when the left constituent was presented as an individual prime (see Footnote 6). Descriptive analyses showed that the priming effect in Experiment 1 (i.e., $17 \mathrm{~ms}$ ) was smaller than that obtained when the left constituent was presented as an individual prime (i.e., $29 \mathrm{~ms}$ ). However, the 12 -ms difference was only marginally significant $(p<.08)$. Thus, an interpretation of the priming effect obtained in Experiment 1 in terms of an additive effect can reasonably be rejected.
} 
Table 2

Reaction Times (RTs; in Milliseconds), Standard Errors of the Means, Percentages of Error, and Eta for Lexical Decisions to Target Words Associated With the Left Constituents of Compound Words With a Transparent or Opaque Right Constituent in Each Condition of Experiment 1, Experiment 4B, and Experiments 1 and $4 B$

\begin{tabular}{|c|c|c|c|c|c|c|c|c|}
\hline \multirow[b]{2}{*}{ Condition } & \multicolumn{4}{|c|}{ Transparent } & \multicolumn{4}{|c|}{ Opaque } \\
\hline & $\mathrm{RT}$ & SEM & $\%$ error & $\eta$ & RT & SEM & $\%$ error & $\eta$ \\
\hline \multicolumn{9}{|c|}{ Experiment $1, N=16\left([\mathrm{C} 1-\mathrm{C} 2]^{\mathrm{a}}\right)$} \\
\hline Related & 492 & 5.5 & 2.2 & .77 & 487 & 5.3 & 2.1 & .10 \\
\hline Unrelated & 509 & 5.3 & 2.8 & & 492 & 5.5 & 1.9 & \\
\hline $\begin{array}{r}\text { Priming } \\
\text { effect }\end{array}$ & $17 *$ & & & & 5 & & & \\
\hline \multicolumn{9}{|c|}{ Experiment $4 \mathrm{~B}, N=16\left([\mathrm{C} 1-\mathrm{C} 2]^{\mathrm{a}}\right)^{\mathrm{b}}$} \\
\hline Related & 521 & 10.5 & 4.8 & .54 & 507 & 9.8 & 3.9 & .04 \\
\hline Unrelated & 541 & 9.0 & 5.8 & & 511 & 7.8 & 4.8 & \\
\hline $\begin{array}{r}\text { Priming } \\
\text { effect }\end{array}$ & $20^{*}$ & & & & 4 & & & \\
\hline
\end{tabular}

Experiments 1 and $4 \mathrm{~B}, N=32$

\begin{tabular}{lcccccccc} 
Related & 507 & 6.4 & 3.5 & .62 & 497 & 5.7 & 3.0 & .06 \\
Unrelated & 524 & 5.8 & 4.3 & & 501 & 5.0 & 3.4 & \\
$\begin{array}{l}\text { Priming } \\
\text { effect }\end{array}$ & $17 *$ & & & & & & & \\
\hline
\end{tabular}

Note. $\mathrm{C} 1=$ left constituent; $\mathrm{C} 2=$ right constituent.

${ }^{a}$ Visual targets appeared in the speech signal. ${ }^{\mathrm{b}}$ Replication of Experiment 1 .

$* p<.05$.

(i.e., the head) was transparent. The absence of a systematic facilitatory priming effect for left constituents suggests that there is no primacy of the left monosyllabic constituents in the access of German compound words. It is interesting that this outcome constitutes a replication of previous findings reported with both a unimodal semantic priming paradigm in Dutch (Sandra, 1990; Zwitserlood, 1994) and a unimodal repetition priming paradigm in Bulgarian (Jarema et al., 1999). Crosslinguistic evidence suggesting that the left constituents of compound words are not systematically activated is fairly discouraging with respect to a decompositional theory postulating that the first syllable of a compound word is an access code for all compound word types (Taft \& Forster, 1976). In Experiment 1, the absence of a priming effect for the left constituents of compound words with an opaque head (i.e., for $\mathrm{O}-\mathrm{O}$ and $\mathrm{T}-\mathrm{O}$ compound words) forces us to refrain from considering the left constituents as an access code to the lexicon. Thus, as German compound words with an opaque head are probably not decomposed, another mode of lexical access has to be postulated to account for their access. A sequential continuous access theory that assumes a direct access to the lexicon that does not depend on the processing of the left constituents could better account for the processing of German compound words with an opaque head.

In contrast, the presence of a facilitatory priming effect for some German compound words rather validates the propositions of the
Taft and Forster (1976) dual-stage search model. However, although a decomposition probably occurs, one has to be careful before concluding that the first constituent of these decomposable compound words is an access code. Indeed, because a priming effect was reported only for the compound words with a transparent head, one can formulate the assumption that the activation of the left constituent depends on the processing of the right constituent but only when the right constituent is transparent. The question of whether the left or the right constituent has primacy for the processing of compound words with a transparent head (i.e., T-T and O-T compound words) is central with respect to the modeling of lexical access to right-headed compound words in Germanic languages. Indeed, the mode of lexical access to compound words would be quite different depending on whether the left or the right constituent has the primacy. The mode of lexical access would be discontinuous (decompositional) but according to a left-to-right mode in one case (primacy of the left constituents) and according to a hierarchical mode in the other case (primacy of the right constituent).

Taken together, the results of Experiment 1 suggest that it is not sufficient to postulate only one mode of lexical access to account for the processing of different types of German compound words. One part of our data is consistent with a decompositional account, and the other part is more in favor of a nondecompositional account. Thus, a cognitive system with two access routes for the types of compound words might be more appropriate in describing the data of Experiment 1. However, before we are able to propose a consistent explanation, we need to test the hypothesis of hierarchical processing for German compound words with a transparent head. Experiment 2 was conducted to test this hypothesis.

\section{Experiment 2}

Experiment 2 was designed to investigate whether the transparent head of German compound words governs the processing of left constituents. To test the assumption that left constituents can be accessed only once the complete lexicosemantic information relative to the transparent head is processed, we examined the activation level of left constituents at a point in the compound words where all lexicosemantic information relative to the head is likely to be still not fully available. Thus, we measured priming effects at the acoustic offset of the left constituents in a crossmodal semantic priming paradigm.

If the activation observed for the left constituents in Experiment 1 was due only to hierarchical processing via the extraction of the transparent heads, a null priming effect should be observed for the compound words with a transparent head in Experiment 2, because here we measured the priming effects before the lexical access of the head was complete. No priming effects would also be predicted for the compound words with an opaque right constituent.

In contrast, if the activation found in Experiment 1 reflects only a residual activation of the left constituents (on-line activation of the access code in a discontinuous left-to-right model), then a priming effect should still be found for compound words with a transparent head. A priming effect would also be expected for compound words with opaque right constituents. 


\section{Method}

Participants. A group of 24 native speakers of German at the University of Leipzig ( 12 men and 12 women; mean age $=25.2$ years, range $=$ 19-31 years) were tested.

Materials, procedure, and design. Materials and procedure were the same as those used in Experiment 1. The present experiment differed from Experiment 1 solely with respect to the target position relative to the prime. Visual targets were presented at the acoustic offset of the left constituent of the auditory primes (for the related, unrelated, and filler pairs). The offset point was determined by visual inspection of the speech signal (the speech wave and spectrograms) and by auditory control.

\section{Results}

We averaged the RTs for correct responses in the eight experimental conditions across participants and items. The results are presented in Table 1.

There was no significant main effect for relatedness, left constituent, right constituent, or word list $\left(F_{\mathrm{S}}<1\right)$. No interaction between the main factors was significant. The number of errors in the related and unrelated conditions ( $4.1 \%$ vs. $4.8 \%$, respectively) did not differ significantly $\left(F_{\mathrm{S}}<1\right)$.

\section{Discussion}

The inability to obtain a semantic priming effect at the acoustic offset of the left constituents, even for the compound words with a transparent head, suggests that the semantic representations of these constituents were not activated on-line. Thus, an interpretation of the priming effect obtained in Experiment 1 in terms of residual activation of the left constituents is disputable. ${ }^{9}$ What clearly emerges from the present data is that in absence of full lexicosemantic information as provided by the head of the compound words, no activation of the left constituents was observed. Accordingly, this pattern of results suggests that the processing of the left constituent could depend on the preliminary extraction of a transparent head. Therefore, the determinant factor in triggering a decomposition would be the transparent right constituent rather than the first syllable of a compound word, at least in spoken word recognition. On the basis of data from Experiments 1 and 2, the idea of a discontinuous hierarchical lexical access for some compound words in German should be considered. However, before reaching firm conclusions about the generality of a decompositional approach for the processing of German compound words with a transparent head, the role of phonetic and prosodic information should be investigated. In fact, the intersyllabic cues of coarticulation and the prosodic structure of the left constituent indicate to the processing system that the sequence to be processed is not a monosyllabic item. Thus, perhaps the left constituent of a compound word is recognized immediately as part of a compound. One may hypothesize that the phonetic and prosodic information signaling a long word could impede the on-line activation of the first constituent. If this is the case, then interpretation of the priming effects found in Experiment 1 in terms of residual activation of the left constituents should be rejected. To test the role of phonetic and prosodic information on the activation level of the left constituents, we conducted Experiment 3.

\section{Experiment 3}

The question we addressed in Experiment 3 was whether the prosodic structure of left constituent and/or the intersyllabic cues of coarticulation between the two constituents inform the processing system that the left constituent is part of a compound. To test this hypothesis, we suppressed the intersyllabic cues of coarticulation between the two constituents of the compound words. For each compound word, both constituents were spoken in isolation and then spliced together, yielding truncated compound words. Moreover, by means of this manipulation, we also controlled the prosodic structure of the primes, resulting in a decomposed prosodic contour. We measured the priming effect at the acoustic offset of the truncated compound words.

If the prosodic and phonetic information signaling a long word was able to impede the activation of the first constituent in Experiment 2, then a priming effect should be obtained for all types of compound words in Experiment 3, because this information is removed. In contrast, if prelexical information does not influence the activation level of left constituents, the same pattern of nul results should be expected in Experiment 3 as in Experiment 2.

\section{Method}

Participants. A group of 32 native speakers of German at the University of Leipzig (16 men and 16 women; mean age $=22.9$ years, range $=$ 22-25 years) were tested.

Material. Truncated compound words were created by splicing together the digital waveforms of complementary monosyllabic left constituents and mono-, di-, or trisyllabic right constituents that were previously recorded in isolation. When the truncated compound words sounded unnatural because of the presence of clicks, we modified the splice through the inspection of waveforms and through auditory inspection. A native German speaker listened to the stimuli to ensure their quality. The stimuli were then corrected until they were considered as natural as possible.

We performed acoustic measures on the primes used in Experiments 1 and 3 to determine whether significant acoustic differences were observable. First, we compared the length (duration in milliseconds) of the left constituents spoken in isolation (Experiment 3 ) with the length of the left constituents embedded in the compound words (Experiment 1). The monosyllabic constituents spoken in isolation were significantly longer $(596 \mathrm{~ms}$, $S E M=8.6)$ than the embedded constituents (489 ms, $S E M=8.2), F_{2}(1$, $135)=92.76, M S E=950.32$. This outcome confirms that syllables produced as monosyllabic words are usually longer than syllables embedded in a polysyllabic word (Klatt, 1976; Lehiste, 1972).

\footnotetext{
${ }^{9}$ Moreover, the absence of a priming effect in Experiment 2 suggests that the priming effects measured in Experiment 1 cannot be attributed to an unexpected semantic association between the compound words and the visual targets. According to the cohort theory (Marslen-Wilson, 1987; Marslen-Wilson \& Welsh, 1978), all the candidate words in the lexicon satisfying an initial alignment with the first $200 \mathrm{~ms}$ of the speech signal are activated. In Experiment 2, one can thus assume that the compound words were already strongly activated in the lexicon and, consequently, would be able to prime semantic associates once the participants decided on the lexical status of the visual targets (on average, $503 \mathrm{~ms}$ after the acoustic onset of the compounds). If the priming effects observed in Experiment 1 were due to the compound words, then the same pattern of results should have been observed in Experiment 2 as that in Experiment 1, because the compound words were also activated in Experiment 2. The null priming effect reported in Experiment 2 clearly indicates that the priming effects observed in Experiment 1 cannot be attributed to the compound words.
} 
Second, we determined the onset and offset fundamental frequency $\left(\mathrm{F}_{0}\right.$; in hertz) for each left constituent spoken in isolation and for each left constituent embedded in the compound words. For each of these critical items, we also determined the peak of fundamental frequency and the duration in milliseconds between the onset of the word and the point in the signal at which the $F_{0}$ peak was found. Statistical analyses showed that all of these acoustic cues did not differ significantly for the two types of items: For the left constituents spoken in isolation, $\mathrm{F}_{0}$ onset $=161 \mathrm{~Hz}, \mathrm{~F}_{0}$ offset $=$ $140 \mathrm{~Hz}$, and $\mathrm{F}_{0}$ peak $=266 \mathrm{~Hz}$; the duration between word onset and $\mathrm{F}_{0}$ peak was $250 \mathrm{~ms}$. For the left constituents embedded in compound words, $\mathrm{F}_{0}$ onset $=149 \mathrm{~Hz}, \mathrm{~F}_{0}$ offset $=163 \mathrm{~Hz}$, and $\mathrm{F}_{0}$ peak $=284 \mathrm{~Hz}$; the duration between word onset and $\mathrm{F}_{0}$ peak was $254 \mathrm{~ms}$. Taken together, the present acoustic measures suggest that duration is the crucial factor in the prosodic structure. Duration allows listeners to discriminate between monomorphemic words spoken in isolation and morphemes embedded in the initial position of polymorphemic words (Cutler et al., 1997; Davis et al., 2002; Grosjean \& Gee, 1987).

The 136 primes in the unrelated condition were created in the same way as the primes in the related condition, that is, by splicing the first monosyllabic constituent (e.g., Schrank [closet]) and the complementary second monosyllabic constituent (e.g., Fach [partition]) to create a truncated control word prime (e.g., Schrankfach [compartment]). The visual targets were the same as those in Experiment 1.

The same additional 408 filler pairs as those in Experiment 1 were used. To avoid auditory contrasts between the three prime sets (experimental, control, and fillers), we also created truncated fillers by splicing together the digital waveforms of complementary monosyllabic left constituents and mono-, di-, or trisyllabic right constituents.

Design and procedure. Procedure and design were the same as those in Experiment 1. Visual probes were presented at the acoustic offset of the truncated compound word primes (for the related, unrelated, and filler pairs).

\section{Results and Discussion}

We subjected the results to the previously designed ANOVA. Relevant data are presented in Table 1.

Mean lexical decision latency was shorter for the related condition $(523 \mathrm{~ms}, S E M=7)$ than for the unrelated condition (535 ms, $S E M=7.8), F_{1}(1,15)=21.40, M S E=224.16$, and $F_{2}(1$, 33) $=3.66, M S E=2,603.56$. The main effects of left constituent and right constituent were significant across participants, $F_{1}(1$, $15)=28.12, M S E=249.82$, and $F_{1}(1,15)=27.34, M S E=$ 445.87; and across items, $F_{2}(1,33)=15.29, M S E=1,037.35$, and $F_{2}(1,33)=26.94, M S E=1,322.30$. No interactions between the main factors were significant. Relatedness had no effect on errors (related $=3.9 \%$, unrelated $=3.9 \% ; F \mathrm{~s}<1)$.

Experiment 3, therefore, provides one clear conclusion: When prelexical information was suppressed, a 12-ms facilitatory priming effect was obtained at the acoustic offset of the whole word, indicating that left constituents representing the prosodic structure of individual words were activated. ${ }^{10}$ The absence of interactions between relatedness and right constituents indicates that the priming effect did not differ significantly for compound words with a transparent head and for compound words with an opaque head; moreover, the absence of an interaction between relatedness, left constituent, and right constituent demonstrates that the priming effect also did not differ significantly in the four categories of compound words. The outcome of Experiment 3 demonstrates that lexical access to left constituents of the four categories of compound words occurred when these constituents reflected the prosodic structure of individual words. In contrast, when constituents reflected the prosodic structure of embedded words, as in Experiment 1 , only left constituents of compound words with a transparent head were processed. In this light, the left constituents of German compound words are accessed under two conditions that allow for decomposition or direct lexical access: (a) when a compound word has a transparent head (Experiment 1), or (b) when left constituents sound like individual words (Experiment 3). The duration of the first morphemes in a compound word in which the head is the final constituent seems to somehow determine the decomposition process.

Our overall results suggest that a transparent final head enables decomposition and that the prosodic information of an initial morpheme certainly steers the cognitive system toward a decompositional route for the processing of compound words. But to investigate more precisely the influence of the head on the activation level of the left constituents, we conducted a last experiment.

\section{Experiment 4}

The purpose of Experiment 4 was to compare the activation level of the left constituent when the head of a compound word was simply suppressed (Experiment $4 \mathrm{~A}$ ) and when it was still available (Experiment 4B, a replication of Experiment 1). By means of a Latin square design, we mixed truncated compound words (e.g., Gast plus white noise; Experiment 4A) and nontruncated compound words (e.g., Gasthaus; Experiment 4B) in four presentation lists.

In Experiment 4A we aimed to examine the activation level of left constituents at the acoustic offset of truncated compound words in which no lexical information relative to the right constituents was available. Thus, we created truncated compound words by replacing right constituents by white noise (only the duration and the amplitude contour of the right constituents remained; e.g., Gast plus white noise). In this way, Experiment 4A created a strong test of whether the priming effect in Experiment 1 was due to the influence of the transparent head on the left constituent or whether it reflected residual activation of the left constituent. By modulating the lexical information relative to the head of the compound word, we could determine whether the activation level of the left constituent varies as a function of this lexical information. Furthermore, measuring the activation level of the left constituent after a delay identical to that in Experiment 1 enabled a test of the duration of the on-line left constituent activation.

If the activation level of the left constituents depends on the processing of the transparent heads of compound words, no priming effect would be expected when the head is replaced by white

\footnotetext{
${ }^{10}$ In contrast, in an additional cross-modal semantic priming experiment conducted in the present study, we failed to measure a priming effect at the acoustic offset of left constituents, even in the absence of coarticulation cues between the two constituents and the prosodic contour of single words. However, on the basis of a higher error rate observed for an early presentation of visual targets (related $=4.7 \%$ and unrelated $=5.3 \%$ ) than for a late presentation (Experiment 1 ; related $=2.1 \%$ and unrelated $=2.4 \%$ ), one can hypothesize that interferences between the visual target and the ongoing auditory compound may have obscured our priming effects.
} 
noise. If this is not the case, results should replicate those of Experiment 1.

The goal of Experiment 4B was to replicate the results obtained in Experiment 1 to test the robustness of the priming effect observed for compound words with a transparent right constituent. We used the same critical material as in Experiment 1.

\section{Method}

Participants. A group of 32 native speakers of German at the University of Leipzig ( 16 men and 16 women; mean age $=23.2$ years, range $=$ 20-28 years) were tested.

Material. Two types of primes were used: truncated compound words and compound words used in Experiment 1. To construct the truncated compound words, we replaced by white noise the 136 right constituents of the 136 compound words used in Experiment 1. This manipulation consisted first of splitting the two constituents and then of applying to the right constituent a transformation algorithm to extract the contour of amplitude only. We then created white noise that was integrated into the contour of amplitude for each right constituent. The resulting stimuli retained some characteristics of the right constituents (i.e., duration and contour of amplitude). Last, we spliced the left constituents and white-noise right constituents back together.

The same 136 compound words and visual targets and the same 408 filler primes as those in Experiment 1 were used. Half the filler primes contained a white-noise right constituent.

Procedure and design. The four presentation lists were designed such that each target was presented under the four priming conditions, but the same targets did not appear in the same list. Each participant performed two presentation lists. To avoid repetition effects by presenting the same visual targets twice, the two experimental sessions had an interval of 1 week. In each list, 136 prime-target pairs were presented: 34 related pairs and 34 unrelated pairs for the truncated compound words and 34 related pairs and 34 unrelated pairs for the compound words. In each list, the 68 related trials and the 68 unrelated trials were pseudorandomly interspersed with the 408 filler pairs (204 truncated compound words and 204 compound words). Thus, each list had $50 \%$ primes with noise and $50 \%$ primes without noise.

\section{Results}

We averaged the RTs for correct responses in the eight experimental conditions across participants and across items separately for Experiments $4 \mathrm{~A}$ and $4 \mathrm{~B}$. Relevant data are presented in Table 1.

We subjected the results of each experiment to a four-way ANOVA in which relatedness (related or unrelated), left constituent (transparent or opaque), and right constituent (transparent or opaque) were considered within-subjects factors and in which word list (List 1, List 2, List 3, or List 4) was considered a between-subjects factor. ${ }^{11}$

Experiment 4A. There were no significant main effects for relatedness, left constituent, right constituent, or word list $\left(F_{\mathrm{s}}<\right.$ $1)$. There were also no significant interactions between the main factors. The number of errors in the related and unrelated conditions $(3.6 \%$ and $4.4 \%$, respectively) did not differ significantly $(F \mathrm{~s}<1)$.

Experiment $4 B$. Mean lexical decision latency was significantly shorter for the related condition $(514 \mathrm{~ms}, S E M=10.8)$ than for the unrelated condition (526 ms, $S E M=12.0$ ) across participants, $F_{1}(1,15)=10.70, M S E=416.32$, and across items, $F_{2}(1$, $33)=5.89, M S E=1,156.58$. There was a significant main effect of left constituent across participants and across items, $F_{1}(1,15)=20.66, M S E=403.53$, and $F_{2}(1,33)=11.39$, $M S E=1,094.10$, indicating that visual targets associated with transparent left constituents were processed slower than those associated with opaque left constituents (528 ms, SEM $=10.3$, vs. $512 \mathrm{~ms}, S E M=11.5$, respectively). Similarly, RTs for the processing of targets were longer when the right constituents were transparent $(531 \mathrm{~ms}, S E M=0.8)$ than when they were opaque $(509 \mathrm{~ms}, S E M=11.3), F_{1}(1,15)=29.42, M S E=542.28$, and $F_{2}(1,33)=26.35, M S E=1,209.88$. The Relatedness $\times$ Right Constituent interaction was significant, $F_{1}(1,15)=4.99, M S E=$ 389.52 , and $F_{2}(1,33)=5.26, M S E=1,079.98$, reflecting significantly shorter RTs in the related than in the unrelated condition but only for the compound words with a transparent head.

Subsequent analyses revealed that only the 20-ms facilitatory priming effect measured for the compound words with a transparent head was significant, $F_{1}(1,15)=17.81, M S E=168.59$, and $F_{2}(1,33)=8.69, M S E=725.98$. This strong effect accounted for $54 \%$ of the variance across participants and for $21 \%$ of the variance across items; in contrast, the small priming effect found for compounds with an opaque head (i.e., $4 \mathrm{~ms}$ ) accounted for only $4 \%$ of the variance across participants and for only $0.1 \%$ of the variance across items. The two facilitatory priming effects (i.e., 20 $\mathrm{ms}$ and $4 \mathrm{~ms}$, respectively; see Table 2) did differ significantly, $F_{1}(1,15)=4.87, M S E=394.73$, and $F_{2}(1,33)=5.17$, $M S E=1,089.45$. Furthermore, relatedness had no effect on errors (related $=4.4 \%$, unrelated $=5.4 \% ; F \mathrm{~s}<1$ ).

Finally, we conducted a subsequent analysis to ensure that the small priming effects observed for compound words with an opaque head (Experiment 1, $5 \mathrm{~ms}$; Experiment 4B, $4 \mathrm{~ms}$ ) did not fail to reach significance because of a lack of power in the experimental designs. For increasing the statistical power, we pooled together complementary data of Experiments 1 and 4B. Then, we ran a new analysis on the pooled data.

A three-way ANOVA with the factors of relatedness (related or unrelated), left constituent (transparent or opaque), and right constituent (transparent or opaque) revealed a significant main effect of relatedness, $F_{1}(1,31)=30.66, M S E=267.52$, and $F_{2}(1$, $33)=16.72, M S E=461.75$, indicating that mean lexical decision latency was significantly shorter for the related condition $(502 \mathrm{~ms}$, $S E M=6.0)$ than for the unrelated condition $(513 \mathrm{~ms}, S E M=5.7)$. There were significant main effects of left constituent, $F_{1}(1$, $31)=15.69, M S E=379.85$, and $F_{2}(1,33)=5.32, M S E=$ 816.22 , as well as of right constituent, $F_{1}(1,31)=46.36, M S E=$ 379.06 , and $F_{2}(1,33)=22.62, M S E=689.20$. The Relatedness $\times$ Right Constituent interaction was significant, $F_{1}(1,31)=10.73$, $M S E=281.18$, and $F_{2}(1,33)=12.94, M S E=364.60$, reflecting shorter RTs in the related than in the unrelated condition but only for the compound words with a transparent head (see Table 2).

Subsequent post hoc tests revealed that only the $17-\mathrm{ms}$ facilitatory priming effect measured for the compound words with a

\footnotetext{
${ }^{11}$ As in Experiment 1, the four-way ANOVA with the factors of relatedness (related or unrelated), left constituent (transparent or opaque), right constituent (transparent or opaque), and syllabic structure of the right constituent (monosyllable or disyllable) failed to show either a main effect of syllabic structure of the right constituent or a Relatedness $\times$ Syllabic Structure of the Right Constituent interaction in Experiment 4B.
} 
transparent head was significant, $F_{1}(1,31)=50.53, M S E=$ 104.02 , and $F_{2}(1,33)=21.55, M S E=291.02$. Descriptive statistics indicated that this priming effect accounted for $62 \%$ of the variance across participants and for $40 \%$ of the variance across items, whereas the nonsignificant priming effect measured for the compound words with an opaque head (i.e., $4 \mathrm{~ms}$ ) accounted for only $6 \%$ of the variance across participants and for only $2 \%$ of the variance across items. The two facilitatory priming effects (i.e., 17 $\mathrm{ms}$ and $4 \mathrm{~ms}$, respectively; see Table 2) differed significantly, $F_{1}(1,31)=10.83, M S E=284.41$, and $F_{2}(1,33)=13.21, M S E=$ 369.42 .

On the basis of this analysis, the hypothesis can be rejected that the facilitatory priming effect observed for compound words with an opaque head failed to reach significance in Experiments 1 and 4B because of a lack of power in the experimental designs. Results of both inferential ( $F$ ratio) and descriptive (eta ratio) statistics converge to demonstrate that this effect is not reliable and is small. Therefore, we concentrate our discussion on the priming effect found for compound words with a transparent head.

\section{Discussion}

Taken together, Experiments 4A and 4B clearly demonstrate that the left constituents of German compound words are activated only when the transparent head of the compound word is processed. The lack of any priming effect when the right constituents were replaced by white noise (Experiment 4A) strengthens the claim that the priming effects observed for the compound words with a transparent head (Experiment 1) cannot be attributed to a residual activation of the left constituents. Experiment 4B, moreover, provides an excellent replication of the pattern of results obtained in Experiment 1, attesting to the robustness of priming for the compound words with a transparent head. The present results strongly indicate that the transparent head of a German compound word is the determining factor in triggering a decomposition of the whole word. On the basis of the data we obtained in the four experiments, we postulate different modes of lexical access for German compound words with transparent and opaque heads. A plausible explanation to account for the present data in German is a processing device in which two routes of processing, a direct route and a decompositional route, work in parallel.

\section{General Discussion}

In the present study, we examined the lexical access to spoken German two-noun compound words. Our manipulation consisted of monitoring semantic priming effects of the left constituents of compound words to test the hypothesis that these constituents could be the access codes to the lexicon. Our results provide a clear picture: There is no primacy of the left constituents for the auditory processing of German compound words. Evidence for priming was observed only for compound words with a transparent right constituent, which in German is the head of the compound word.

Experiment 1 demonstrated that left constituents of compound words can prime their semantic associates, but this is true only for compound words with a transparent head. This finding suggests that the semantic transparency of the right constituents influences the activation level of the left constituents. In contrast, priming effects were not obtained at the acoustic offset of left constituents
(Experiment 2), suggesting that the priming effects found in Experiment 1 could not be attributed to residual on-line activation of left constituents. Furthermore, the generalized priming effects obtained in Experiment 3 at the acoustic offset of compound words with a decomposed prosodic contour suggest that left constituents with the duration of single words are accessed regardless of the transparency of the head. The replication in Experiment $4 \mathrm{~B}$ of the priming effects for compound words with transparent heads found in Experiment 1 greatly strengthened our assumption that only German compound words with a transparent head are decomposed and that transparent heads are the access code to the lexicon. A possible interpretation of the robust priming effects in terms of residual activation could definitively be rejected on the basis of findings collected in Experiment 4A; here, we demonstrated no activation of the left constituents after a delay equivalent to the duration of the right constituents that were white noise. ${ }^{12}$

The priming effects observed only for compound words with a transparent head may be accounted for by postulating a processing system in which these words are accessed via the preliminary extraction of their head, which is used as an access code to the lexicon. Conversely, compound words with an opaque head are identified by a whole-word process. Because neither nonparallel models (the stem allomorph/inflectional decomposition [SAID] model, Niemi et al., 1994) nor parallel models (the augmented addressed morphology [AAM] model, Caramazza et al., 1988; the morphological race model, Frauenfelder \& Schreuder, 1992; the meta-model, Schreuder \& Baayen, 1995) seem to be able to account for the strong distinction emerging from our data, we formulated the following explanation.

\section{A Prosody-Assisted Processing Device}

The prosody-assisted processing device for lexical access assumes two routes, a direct and a decompositional route, that work in parallel. The process of lexical access for lexicalized compound words in such a dual-route model consists of both a lexical retrieval process and a morphological decompositional process that are accomplished in two processing stages. Such a cognitive system can be described as follows.

During a first stage of processing, a left-to-right continuous analysis is completed at the acoustic-phonetic level. The processing system automatically searches for the activation of a lexical unit at the word level by continuously mapping the ongoing acoustic-phonetic information onto stored lexical representations using a direct route. At the same time, it also analyzes the prosodic structure of the first morpheme on the basis of its duration, as this is a crucial prosodic cue for determining whether the first morpheme is the onset of a compound word or whether it represents a separate monomorphemic word. The output of prosodic analysis is a determining factor in setting the functional configuration of the processing system. If this output indicates a noncompound word (longer duration of the first morpheme), the processing system continues to match the acoustic-phonetic input with the word

\footnotetext{
${ }^{12}$ In French, Isel and Bacri (1999, Experiment 1) also showed that the semantic form of initial monosyllabic words embedded in disyllabic monomorphemic words (e.g., car [bus] in cargo [freighter]) was not activated at the acoustic offset of the disyllables.
} 
detectors at the word level, until the appropriate detector reaches the threshold for best match. In this case, the processing system has set a monomorphemic route configuration. In contrast, if the output of the prosodic analysis informs the processor that the first morpheme is the onset of a compound word, then the processing system activates a decompositional route in addition to the direct route already activated at the onset of the speech input (a dualroute configuration). The decompositional route extracts the right constituent during a first substage of processing and maps this constituent onto morphological units stored in a morphological sublexical level during a second substage of processing. ${ }^{13}$ Thus, in this case, both direct and decompositional routes are working in parallel. Because the involvement of the routes occurs sequentially, such a processing system could be described as a cascading parallel model.

During a second stage of processing, lexical access is completed for both routes, but the mode of access differs with respect to the route. For the direct route, the word level is directly accessed and a successful recognition is completed when the activation level of the appropriate representation reaches the criterion for best match. In contrast, for the decompositional route, the sine quo non condition of access to the word level is the preliminary activation of a morphological unit (i.e., an access code) at a morphological sublexical level. First, each route has to be considered as a potentially appropriate route. The appropriateness of a route per default emerges as the result of successful access to the lexicon. Once the appropriate route emerges, the other route is automatically disregarded. Thus, compound words with an opaque head are usually identified by a whole word process, whereas compound words with a transparent head have to be decomposed prior to the activation of their whole-word representation at the word level.

\section{Importance of the Head in German Compound Words}

At first glance, the idea of hierarchical lexical access for compound words with a transparent head may be surprising, especially given the inherent left-to-right nature of the speech signal. Consider, however, that the right constituents of compound words in Germanic languages contain the most important information with respect to the whole word. For example, the head determines general syntactic properties, such as syntactic gender of the whole word, and for some categories of compound words, the head also specifies to what kind of thing a compound word refers. Moreover, given that prosodic information concerning the morphemic structure (mono- vs. polymorphemic word) is already available early in the processing of the first constituent, it is plausible that this information delays access to semantic properties of the first constituent while waiting for the second constituent to be processed. Consequently, in Germanic languages in which compound words are extremely common, the processing system has been designed to wait for the final segment of a compound word to be able to extract crucial information for the whole word. It is thus not untenable to postulate a decompositional route that uses the most informative segment of the word, the head, as the determining factor in decomposition.

\section{Conclusions}

The present investigation revealed that German compound words with a transparent final head are decomposed, whereas
German compound words with an opaque head are usually not. The processing system seems to use two separate modes to access German compound words: a direct route for compound words with an opaque head or a decompositional route for compound words with a transparent head. We suggest that for auditorily presented compound words, the prosodic structure (i.e., the duration) of initial morphemes is able to assist the processing system in activating a decompositional route at the offset of these morphemes. Therefore, we propose a new factor relevant for a dual-route cognitive system in the auditory processing of words, namely prosody. We conclude that for known German compound words, a prosody-assisted processing device consisting of a direct route and a decompositional route might be suitable to account for the data from the four experiments we conducted. Further investigations using novel T-T compound words, compound words in other languages, and compound words presented visually will be necessary before understanding the functionality of such a prosodyassisted cognitive system.

${ }^{13}$ The extraction of the first morpheme follows the successful extraction of the second morpheme.

\section{References}

Andrews, S. (1986). Morphological influences on lexical access: Lexical or nonlexical effects. Journal of Memory and Language, 25, 726-740.

Baayen, R. H., Piepenbrock, R., \& Gulikers, L. (1995). The CELEX lexical database [Computer software]. Philadelphia, PA: Linguistics Data Consortium, University of Pennsylvania.

Butterworth, B. (1983). Lexical representation. In B. Butterworth (Ed.), Language production: Vol. 2. Development, writing and other language processes (pp. 257-294). San Diego, CA: Academic Press.

Caramazza, A., Laudanna, A., \& Romani, C. (1988). Lexical access and inflectional morphology. Cognition, 28, 297-332.

Collins, A. M., \& Loftus, E. F. (1975). A spreading activation theory of semantic processing. Psychological Review, 82, 407-428.

Cutler, A., Dahan, D., \& van Donselaar, W. (1997). Prosody in the comprehension of spoken language: A literature review. Language and Speech, 40, 141-201.

Davis, M. H., Marslen-Wilson, W. D., \& Gaskell, M. G. (2002). Leading up the lexical garden path: Segmentation and ambiguity in spoken word recognition. Journal of Experimental Psychology: Human Perception and Performance, 28, 218-244.

Frauenfelder, U. H., \& Schreuder, R. (1992). Constraining psycholinguistic models of morphological processing and representation: The role of productivity. In G. Booij \& J. Van Marle (Eds.), Yearbook of morphology (pp. 165-183). Dordrecht, the Netherlands: Kluwer Academic.

Frost, R., Deutsch, A., \& Forster, K. I. (2000). Decomposing morphologically complex words in a nonlinear morphology. Journal of Experimental Psychology: Learning, Memory, and Cognition, 26, 751-765.

Grosjean, F., \& Gee, J. (1987). Prosodic structure and spoken word recognition. Cognition, 25, 135-155.

Hyönä, J., \& Pollatsek, A. (1998). Reading Finnish compound words: Eye fixations are affected by component morphemes. Journal of Experimental Psychology: Human Perception and Performance, 24, 1612-1627.

Inhoff, A. W., Radach, R., \& Heller, D. (2000). Complex compounds in German: Interword spaces facilitate segmentation but hinder assignment of meaning. Journal of Memory and Language, 42, 23-50.

Isel, F., \& Bacri, N. (1999). Spoken-word recognition: The access to embedded words. Brain \& Language, 68, 61-67.

Jarema, G., Busson, C., Nikolova, R., Tsapkini, K., \& Libben, G. (1999). 
Processing compounds: A cross-linguistic study. Brain \& Language, 68, 362-369.

Kehayia, E., Jarema, G., Tsapkini, K., Perlak, D., Ralli, A., \& Kadzielawa, D. (1999). The role of morphological structure in the processing of compounds: The interface between linguistics and psycholinguistics. Brain \& Language, 68, 370-377.

Klatt, D. (1976). Linguistic uses of segmental duration in English: Acoustic and perceptual evidence. Journal of the Acoustical Society of America, 59, 1208-1221.

Laudanna, A., Badecker, W., \& Caramazza, A. (1989). Priming homographic stems. Journal of Memory and Language, 28, 531-546.

Lehiste, I. (1972). The timing of utterances and linguistic boundaries. Journal of the Acoustical Society of America, 51, 2018-2024.

Libben, G. (1994). How is morphological decomposition achieved? Language and Cognitive Processes, 9, 369-391.

Lima, S. D., \& Pollatsek, A. (1983). Lexical access via an orthographic code? The basic orthographic syllable (BOSS) reconsidered. Journal of Verbal Learning and Verbal Behavior, 22, 310-322.

Marslen-Wilson, W. D. (1987). Functional parallelism in spoken word recognition. Cognition, 25, 71-102.

Marslen-Wilson, W. D., \& Welsh, A. (1978). Processing interactions and lexical access during word recognition in continuous speech. Cognitive Psychology, 10, 29-63.

Monsell, S. (1985). Repetition and the lexicon. In A. W. Ellis (Ed.), Progress in the psychology of language (Vol. 2, pp. 147-195). Hove, England: Erlbaum.

Niemi, J., Laine, M., \& Tuominen, J. (1994). Cognitive morphology in Finnish: Foundations of a new model. Language and Cognitive Processes, 9, 423-446.

Pollatsek, A., Hyönä, J., \& Bertram, R. (2000). The role of morphological constituents in reading Finnish compound words. Journal of Experimental Psychology: Human Perception and Performance, 26, 820-833.
Sandra, D. (1990). On the representation and processing of compound words: Automatic access to constituent morphemes does not occur. Quarterly Journal of Experimental Psychology: Human Experimental Psychology, 42(A), 529-567.

Schreuder, R., \& Baayen, R. H. (1995). Modeling morphological processing. In L. B. Feldman (Ed.), Morphological aspects of language processing (pp. 131-154). Hillsdale, NJ: Erlbaum.

Slowiaczek, L. M., Nusbaum, H. C., \& Pisoni, D. B. (1987). Phonological priming in auditory word recognition. Journal of Experimental Psychology: Learning, Memory, and Cognition, 13, 64-75.

Tabossi, P. (1996). Cross-modal semantic priming. Language and Cognitive Processes, 11, 553-699.

Taft, M. (1979). Lexical access via an orthographic code: The basic orthographic syllabic structure (BOSS). Journal of Verbal Learning and Verbal Behavior, 18, 21-39.

Taft, M., \& Forster, K. I. (1975). Lexical storage and retrieval of prefixed words. Journal of Verbal Learning and Verbal Behavior, 14, 638-647.

Taft, M., \& Forster, K. I. (1976). Lexical storage and retrieval of polymorphemic and polysyllabic words. Journal of Verbal Learning and Verbal Behavior, 15, 607-620.

Zhou, X., \& Marslen-Wilson, W. D. (1994). Words, constituents, and syllables in the Chinese mental lexicon. Language and Cognitive Processes, 9, 393-422.

Zwitserlood, P. (1994). The role of semantic transparency in the processing and representation of Dutch compound words. Language and Cognitive Processes, 9, 341-368.

Received September 12, 2001

Revision received September 18, 2002

Accepted September 24, 2002 